destruction of thalamocapsular nucleus revealed slight decrease of motor activity, however, simultaneously it caused the remarkable improvement of tremor or rigidity. But in some cases there was a reappearance of the same symptom at the second week after operation. The results by long observation are also reported.

At the present stage, the effects of stereotaxic encephalotomy on Parkinsonism are not sufficient but the combination of surgical treatment with suitable medicament therapy may make it possible to obtain the better results.

\title{
10. Stimulation and Destruction of the Hypothalamus
}

\author{
Keiji Sano, Masumi Yoshioka and Motohide Ogashiwa \\ Dept. of Neurosurgery, School of Medicine, University of Tokyo
}

We have performed the postero-medial hypothalamotomy, namely stereotaxic electrocoagulation of the ergotropic zone of the hypothalamus, as treatment of disorders of emotion as manifested in aggressive behaviors or rage attacks. This procedure produced most marked sedative effects and less side effects. The region of the hypothalamus to be cauterized by this procedure is, in lateral view, the triangular area bounded by the lines connecting the midpoint of the AC-PC line, the rostral pole of the mammillary body and the rostral end of the aqueduct, and is, in antero-posterior view, from one to three $\mathrm{mm}$. lateral to the lateral wall of the third ventricle.

During this operation we recorded electrographic changes by means of the needle inserted into the hypothalamus and implanted electrodes in the hippocampus, the amygdala and the frontal and temporal cortical areas. In these recordings, there were some cases in which a close connection between the postero-medial hypothalamus and the hippocampus was clearly noted.

In animal experiments, we used cats with chronically implanted electrodes in the preoptic region, the posterior hypothalamus, the reticular formation, the amygdala, the hippocampus and the sensory-motor area of the neocortex, in order to observe both electrographic changes of the neo-, paleo- and archicortex, and concomitant behavioral changes.

After destruction of the unilateral preoptic area, disappearance of the so-called hippocampal arousal waves and dissociation between the electrical activities of the amygdala and the neocortex were observed; however, no remarkable behavioral changes were noted.

In case of bilateral destruction of the preoptic area, the cat exhibited rage, pronounced being irritable and hyperactive, possibly due to dominance of the 
ergotropic circuits.

After destruction of the unilateral posterior hypothalamus, the hippocampal arousal waves disappeared and the cat became hypoactive and somnolent. After bilateral destruction of the posterior hypothalamus, the hypoactive and somnolent states were remarkably increased and deep sleep patterns were observed in electrography. These changes could be interpreted as a state of dominance of the trophotropic circuits.

\title{
11. Stereotaxic CEM-Thalamotomy for Relief of Intractable Visceral Pain \\ - Experimental Bases and Clinical Report -
}

\author{
Miyoshi Urabe, Takashi Tsubokawa, Hiraki SakuraI, Makoto Kikuchi, \\ Sadamitsu MukaI, Satoru Kadoya, Yukio SeKI, \\ Yoh WATANABE and Noboru HaMabe
}

Dept. of Surgery (I), School of Medicine, University of Kanazawa

The stereotaxic thalamotomy have not yet found the acceptance of corresponding procedures for the relief of extrapyramidal motor disorder, although lesions have been produced for the treatment in the nucleus dorsomedialis (Spiegel \& Wycis), the nucleus ventralis posterior (Talairach), nucleus ventralis caudalis parvocellularis (Reichert et al.) and the posterior thalamic area (Mark et al.).

The failure or the difficulty to relief intractable pain by means of thalamotomy was caused by the complicated pain recepting system in the thalamus and tis function.

Our experimental data on the inhibitory and facilitatory mechanisms of the splanchnic afferent impulses in the central nervous system showed that the recepting mechanisms of visceral afferent impulses in the nucleus centrum medianum (CEM) were very differ from those of the nucleus ventralis posterior, the mesencephalon and the spinal cord. The following facts were recognized in the CEM: 1) there are convergence neurons between the viscerosensory and somatosensory fibers, 2) the evoked potential elicited splanchnic stimulation does not follow $10 / \mathrm{sec}$ stimuli and does not show P.T.P., 3) spindle bursts $(25 \sim 27 / \mathrm{sec})$ are produced in a state of appearance of P.T.P. following high frequency stimulation of splanchnic nerve, and 4) these spindle bursts are also produced by cortical strychnization. On the other hand, unital action potentials on the mesencephalic reticular formation elicited by noxious stimuli are facilitated by the stimulation of the CEM $(10 \sim 100 / \mathrm{sec})$. 